\title{
Effects of oral cystine and glutamine on exercise-induced changes in gastrointestinal permeability and damage markers in young men
}

\author{
Yusei Tataka ${ }^{1}$ Miki Haramura ${ }^{2}$. Yuka Hamada ${ }^{2} \cdot$ Miho Ono $^{3}$ - Sakiko Toyoda ${ }^{4}$ Toshiyuki Yamada ${ }^{5}$. Ayano Hiratsu ${ }^{1}$. \\ Katsuhiko Suzuki ${ }^{6} \cdot$ Masashi Miyashita $^{6}$ (D)
}

Received: 4 July 2021 / Accepted: 11 January 2022 / Published online: 1 February 2022

(c) The Author(s) 2022

\begin{abstract}
Purpose Although acute prolonged strenuous exercise has been shown to increase markers of gastrointestinal permeability and damage, little is known regarding the efficacy of nutritional supplement interventions on the attenuation of exerciseinduced gastrointestinal syndrome. This study addressed the effects of oral amino acid supplementation on markers of gastrointestinal permeability and damage in response to exercise.

Methods Sixteen active men aged $22.7 \pm 2.6$ years (mean \pm standard deviation) completed placebo or cystine and glutamine supplementation trials in random order. Participants received either a placebo or cystine and glutamine supplements, three times a day for 5 days, separated by a 2 -week washout period. On day 6 , participants took their designated supplements $30 \mathrm{~min}$ before running at a speed corresponding to $75 \%$ of maximal oxygen uptake for $1 \mathrm{~h}$, followed by a 4 -h rest period. Blood samples were collected pre-exercise, immediately post-exercise, 30 min post-exercise, and 1, 2 and $4 \mathrm{~h}$ post-exercise on day 6. The plasma lactulose to mannitol ratio (L:M) and plasma intestinal fatty acid-binding protein (I-FABP) were used as markers of gastrointestinal permeability and damage, respectively.

Results Plasma L:M (linear mixed model, coefficient \pm standard error: $-0.011 \pm 0.004, P=0.0090$ ) and changes (i.e., from pre-exercise) in plasma I-FABP (linear mixed model, $-195.3 \pm 65.7$ coefficient \pm standard error $(\mathrm{pg} / \mathrm{mL}), P=0.0035)$ were lower in the cystine and glutamine supplementation trial than in the placebo trial.

Conclusion Oral cystine and glutamine supplementation attenuated the markers of gastrointestinal permeability and damage after $1 \mathrm{~h}$ of strenuous running in young men.

Trial registration number UMIN000026008.

Date of registration 13 December 2018.
\end{abstract}

Keywords Exercise-induced gastrointestinal syndrome $\cdot$ Amino acids supplementation $\cdot$ Strenuous running .

Lactulose:mannitol $\cdot$ Intestinal fatty acid-binding protein

Masashi Miyashita

m.miyashita@waseda.jp

1 Graduate School of Sport Sciences, Waseda University, Saitama, Japan

2 Waseda Institute for Sport Sciences, Waseda University, Saitama, Japan

3 Institute of Food Sciences and Technologies, Ajinomoto Co., Inc., Kanagawa, Japan

4 Research Institute for Bioscience Products \& Fine Chemicals, Ajinomoto Co., Inc., Kanagawa, Japan

5 Sports Nutrition Department, Ajinomoto Co., Inc., Tokyo, Japan

6 Faculty of Sport Sciences, Waseda University, 2-579-15 Mikajima, Tokorozawa, Saitama 359-1192, Japan

$\begin{array}{ll}\text { Abbreviations } \\ \text { Cys2/Gln } & \text { Cystine and glutamine supplementation } \\ \text { HDL-C } & \text { High-density lipoprotein cholesterol } \\ \text { I-FABP } & \text { Intestinal fatty acid-binding protein } \\ \text { LDL-C } & \text { Low-density lipoprotein cholesterol } \\ \text { L:M } & \text { Lactulose to mannitol } \\ \text { NEFA } & \text { Non-esterified fatty acids } \\ \text { SD } & \text { Standard deviation } \\ \text { SE } & \text { Standard error } \\ \text { TC } & \text { Total cholesterol } \\ \text { TG } & \text { Triglycerides }\end{array}$

Abbreviations

Cys2/Gln Cystine and glutamine supplementation

HDL-C High-density lipoprotein cholesterol

I-FABP Intestinal fatty acid-binding protein

LDL-C Low-density lipoprotein cholesterol

L:M Lactulose to mannitol

NEFA Non-esterified fatty acids

SD Standard deviation

SE Standard error

TG Triglycerides 


\section{Introduction}

An acute bout of strenuous exercise has been shown to increase gastrointestinal permeability and intestinal cellular damage, attenuating barrier function and this leads to an initial pro-inflammatory cascade, eventually causing gastrointestinal distress [1-3]. These exercise-induced increases in gastrointestinal permeability and intestinal cellular damage are thought to be mediated via the following two main mechanisms: (1) an increase in core temperature during or shortly after strenuous exercise and/or (2) reduced total splanchnic perfusion, causing gastrointestinal ischemia [4]. Although these changes in gastrointestinal permeability and intestinal cellular damage are transient, it is important to address the chronic consequences of repeated bouts of training sessions that lead to chronic health complications, including chronic inflammatory conditions and fatigue [5].

Some nutritional supplement intervention studies, including bovine colostrum, polyphenols, probiotics, and amino acids have examined the effects of supplementation on exercise-induced increases in gastrointestinal permeability and intestinal cellular damage (for a review of these, see Reference [5]). For instance, glutamine (an amino acid) supplementation has been shown to reduce increased gastrointestinal permeability and intestinal cellular damage from strenuous exercise [6-9]. In addition, cystine (a sulphur-containing amino acid) is the main precursor of glutathione [10], which protects the cells against oxidative stress and is known to inhibit inflammation [11, 12]. Indeed, a recent in vitro study demonstrated that cystine reduced oxidative stress-induced intestinal barrier dysfunction [13]. Collectively, these findings indicate that glutamine and cystine may play important roles in protecting intestinal barrier function via heat and oxidative stress-induced signaling pathways, respectively. However, it remains unknown whether combined glutamine and cystine supplementation can effectively attenuate the exercise-induced increase in gastrointestinal permeability and damage in humans.

Therefore, the purpose of the present study was to examine the effects of oral cystine and glutamine supplementation on markers of gastrointestinal permeability and damage in response to an acute bout of prolonged strenuous exercise in active men. We chose the plasma lactulose to mannitol (L:M) ratio and plasma intestinal fatty acidbinding protein (I-FABP) as markers of gastrointestinal permeability and mucosal damage, respectively. Plasma L:M ratio, not urine L:M ratio, was chosen since the collection of incomplete or inaccurately timed urine samples may lead to errors in estimation of prescribed sugar permeability. Plasma I-FABP was chosen since this is widely used in the previous studies and it reflects a breakdown in endothelial cell integrity (for a review, see Reference [3]). We tested the hypothesis that compared to ingestion of maltodextrin, oral cystine and glutamine supplementation would attenuate exercise-induced increases in plasma L:M and I-FABP concentrations.

\section{Materials and methods}

\section{Participants}

After approval from the Institutional Ethical Advisory Committee (Approval number: 2018-270), 16 young, Japanese men gave written informed consent to participate in this study. This study was registered in advance using the University Hospital Medical Information Network Center, a system for registering clinical trials (ID: UMIN000026008). Exclusion criteria were as fllows: being a current smoker; taking any medication or supplement known to affect lipid and carbohydrate metabolism; major illness, including gastrointestinal disorders or physical problems (acute or chronic), limiting the ability to perform physical activity. Habitual information regarding physical activity were obtained via interviews, while additional questionnaires indicated that all participants met the current international public health guidelines for physical activity (i.e., moderateintensity physical activity for at least $150 \mathrm{~min}$ per week, vigorous-intensity physical activity for $75 \mathrm{~min}$ per week or an equivalent combination of moderate and vigorous-intensity physical activity) [14]. The physical characteristics of the participants (mean \pm standard deviation $(\mathrm{SD})$ ) were as follows: age, $22.7 \pm 2.6$ years; height, $1.71 \pm 0.05 \mathrm{~m}$; body mass, $67.0 \pm 8.0 \mathrm{~kg}$; body mass index, $22.8 \pm 2.5 \mathrm{~kg} / \mathrm{m}^{2}$; and maximum oxygen uptake (measured while running on a treadmill) $54.3 \pm 8.8 \mathrm{~mL} / \mathrm{kg} / \mathrm{min}$.

\section{Anthropometry}

Body mass was measured to the nearest $0.1 \mathrm{~kg}$ using a digital scale (Inner Scan 50, Tanita Corporation, Tokyo, Japan). Height was measured to the nearest $0.1 \mathrm{~cm}$ using a stadiometer (YS-OA, AS One Corporation, Osaka, Japan). Body mass index was calculated as weight in kilogrammes divided by the square of height in metres.

\section{Preliminary tests}

Approximately 14 days before the first main trial, participants underwent two preliminary exercise tests performed on a motorized treadmill (Jog Now 700, Technogym, Cesena, Italy). A 16-min, four-stage, submaximal treadmill test was conducted to determine the 
relationship between running speed and oxygen uptake. Initial running speed was set between 6.0 and $8.0 \mathrm{~km} / \mathrm{h}$ depending on each participant's physical activity level (as determined by the initial screening interview and questionnaire). The treadmill was level throughout the test period, and speed was increased by 1.0 or $1.5 \mathrm{~km} / \mathrm{h}$ every $4 \mathrm{~min}$, depending on each participant's physical activity level. Next, maximum oxygen uptake was directly measured using an incremental uphill protocol at a constant speed, until participants reached volitional fatigue [15]. The initial inclination of the treadmill was set at $3.5 \%$ for the test. Thereafter, the gradient was increased by $2.5 \%$ every $3 \mathrm{~min}$. Heart rate was monitored throughout these tests using short-range telemetry (Polar RCX3, Polar Electro, Kempele, Finland). Oxygen uptake, carbon dioxide production and respiratory exchange ratio were measured using a stationary gas analyzer (Quark RMR, COSMED, Rome, Italy). Ratings of perceived exertion were periodically assessed during tests using the Borg scale [16]. Data generated from these two tests were used to determine the running workload (i.e., running speed) corresponding to $75 \%$ of each participant's maximum oxygen uptake, and this workload was used for the main trials.

Seven days after completing the preliminary exercise tests, one baseline gastrointestinal permeability evaluation was performed (further details are provided under "Evaluation of gastrointestinal permeability"). This baseline evaluation was conducted in a resting state (i.e., without performing exercise) throughout the 6-h evaluation period.

\section{Study design and protocol}

A randomized, double-blind, placebo-controlled crossover design was used in the present study. The primary outcomes of the present study were plasma L:M and I-FABP concentrations. Each participant underwent two, 6-day trials in a randomized, counterbalanced order: placebo or cystine and glutamine supplementation. The interval between trials was 14-22 days. A schematic illustration of the study protocol is presented in Fig. 1. Participants ingested $0.23 \mathrm{~g}$ of L-cystine, $1.00 \mathrm{~g}$ of L-glutamine and $1.23 \mathrm{~g}$ of maltodextrin three times (i.e., at 1000,1500 and before sleep) per day for 5 days or $2.46 \mathrm{~g}$ of maltodextrin three times per day (i.e., at 1000 , 1500 and before sleep) for 5 days. This glutamine dose and regimen were chosen since previous studies observed a suppressive effect of glutamine on oxidative stress [17] and no adverse effects in healthy adults when the individuals consumed a higher dose of glutamine than the present study [6]. Also, this cystine dose and regimen were chosen because of its high safety profile and no adverse effects in healthy adults (i.e., $2100 \mathrm{mg} /$ day for 4 weeks) [18]. Participants were instructed to maintain their current physical activity level during the 5-day supplementation period and to refrain from taking any medications or supplements on days 3, 4 and 5 . Participants were also instructed to refrain from exercising and to consume three standardized meals (i.e., provided by investigators) on day 5. Furthermore, all participants were informed of the foodstuffs (fruits, fruit juice, dairy products, chocolate, honey, mushrooms and chewing gum) that affect the gastrointestinal permeability evaluation before the main trials and they were asked to avoid consuming these food items on day 5 in the present study. On the morning of day

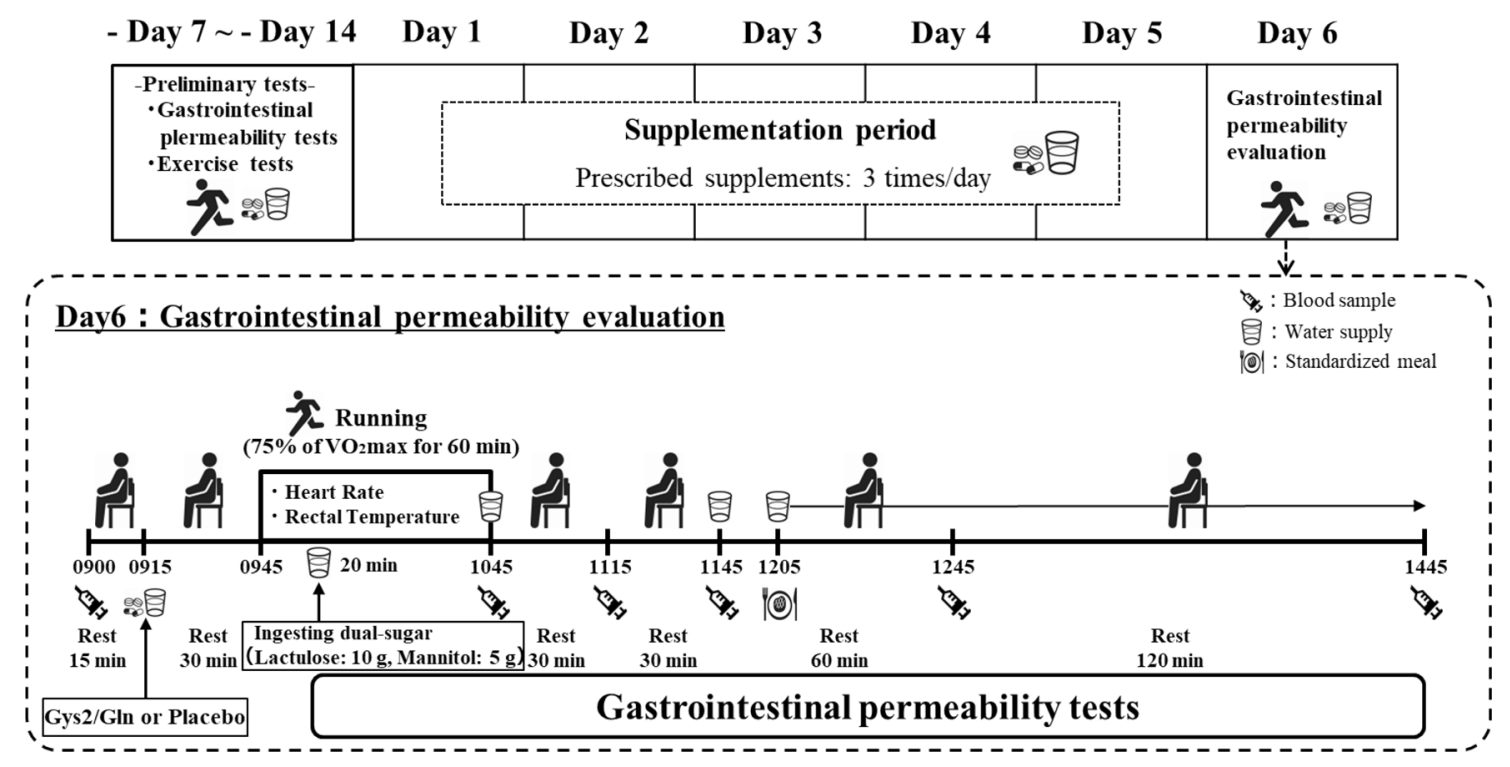

Fig. 1 A schematic representation of the study schedule and protocol. VO2max maximum oxygen uptake, Cys2/Gln cystine and glutamine supplementation 
6, participants reported to the laboratory at 0845 after a $12-\mathrm{h}$ overnight fast (no food or drink after 2100 on day 5) in both trials. No drinking water was permitted after 0645 on the morning of day 6 in either trial. Drinking water and a standardized meal was provided to each participant during a 6-h laboratory-based experiment on day 6 and this was standardized between trials as it affects gastrointestinal permeability evaluations (further details are provided under "Evaluation of gastrointestinal permeability"). Body mass was measured, and a rectal probe was inserted to measure the rectal temperature during exercise using a thermistor $(401 \mathrm{~J}$, Nikkiso-Therm Co., Ltd., Tokyo, Japan). After 15 min, a fasting venous blood sample was collected in a seated position by venepuncture at 0900 . Further venous blood samples were collected immediately post-exercise (1045), and $30 \mathrm{~min}$ (1115), $1 \mathrm{~h}$ (1145), $2 \mathrm{~h}$ (1245) and $4 \mathrm{~h}$ (1445) post-exercise. 15 min after collecting fasting blood samples (at 0915), participants remained seated in a chair and ingested their designated supplements [i.e., placebo (2.46 g of maltodextrin) or cystine and glutamine $(0.23 \mathrm{~g}$ of L-cystine, $1.00 \mathrm{~g}$ of L-glutamine, and $1.23 \mathrm{~g}$ of maltodextrin)] with $200 \mathrm{~mL}$ of warm water $\left(37^{\circ} \mathrm{C}\right)$. After $30 \mathrm{~min}$ of rest, participants performed running exercises at a speed corresponding to $75 \%$ of maximal oxygen uptake for $1 \mathrm{~h}$. The atmospheric temperature and relative humidity during the 1 -h exercise session was maintained at $25{ }^{\circ} \mathrm{C}$ and $60 \%$, respectively, in both trials. Heart rate was monitored throughout exercise using short-range telemetry (Polar RCX3, Polar Electro, Kempele, Finland), and ratings of perceived exertion were periodically assessed [16]. Thereafter, participants were asked to remain seated (reading, writing, or working on a computer) in the laboratory until 1445 . The mean temperature and humidity during the experimental trials were $25.4 \pm 1.1^{\circ} \mathrm{C}$ and $56.3 \pm 3.9 \%($ mean $\pm \mathrm{SD})$, respectively.

\section{Evaluation of gastrointestinal permeability}

Gastrointestinal permeability was evaluated by analysing the plasma samples using the liquid chromatography-tandem mass spectrometry method based on previously published methods [19]. Participants consumed a standardized, dual-sugar solution containing $10 \mathrm{~g}$ of lactulose and $5 \mathrm{~g}$ of mannitol in $100 \mathrm{~mL}$ of warm water $\left(37^{\circ} \mathrm{C}\right)$ and $200 \mathrm{~mL}$ of warm water $\left(37^{\circ} \mathrm{C}\right) 20 \mathrm{~min}$ after the start of running in each exercise trial in the main experiment (1005) or rest (i.e., the baseline gastrointestinal permeability evaluation) in the preliminary test. Participants were asked to ingest $200 \mathrm{~mL}$ of warm water $\left(37^{\circ} \mathrm{C}\right)$ immediately post-exercise (1045) and $1 \mathrm{~h}$ post-exercise (1145). Thereafter, participants were asked to ingest $500 \mathrm{~mL}$ of room temperature water $\left(25^{\circ} \mathrm{C}\right)$, and this volume was asked to be consumed until the end of each main trial (1445). The timing and volumes of ingestion mimicked the baseline (i.e., resting) gastrointestinal permeability evaluation conducted in the preliminary test. The plasma samples were deproteinized with acetonitrile after adding stable isotope labels as internal standard substances. The prepared samples were quantitated using ultra-high performance liquid chromatography with electrospray ionization tandem mass spectrometry (Nexera, Shimadzu Co., Kyoto, Japan; QTRAP 5500, AB Sciex Pte. Ltd., Tokyo, Japan). The analysis method was validated concerning the bioanalytical method validation guidelines [20]. The intra-assay coefficients of variation were $9.3 \%$ for lactulose and $10.4 \%$ for mannitol. The validity of the measured values was confirmed by the quality control sample, and the criteria were $100 \pm 15 \%$.

\section{Evaluation of gastrointestinal damage}

Plasma I-FABP concentrations were determined using EDTA plasma via enzyme-linked immunosorbent assay kits (HK406, Hycult Biotech Inc., Pennsylvania, USA). All analyses for each participant were completed within the same run for each measure. The intra-assay coefficient of variation was $4.4 \%$. We planned to analyze absolute values. However, since there was a large inter-participant variability in plasma I-FABP in the present study as was the case in a previous study [21], we presented plasma I-FABP concentration as changes compared with fasting (0900) values.

\section{Other blood analyses}

For fasting serum triglycerides (TG), non-esterified fatty acids (NEFA), total cholesterol (TC), low-density lipoprotein cholesterol (LDL-C) and high-density lipoprotein cholesterol (HDL-C) measurements, venous blood samples were collected into tubes containing clotting activators to isolate serum. Thereafter, samples were allowed to clot for $30 \mathrm{~min}$ at room temperature (i.e., $25^{\circ} \mathrm{C}$ ) and were centrifuged at $1861 \times g$ for $10 \mathrm{~min}$ at $4{ }^{\circ} \mathrm{C}$. Serum was removed, aliquoted and stored at $-80^{\circ} \mathrm{C}$ for later analysis. For fasting plasma glucose measurements, venous blood samples were collected in tubes containing sodium fluoride-EDTA. Thereafter, both tubes were immediately centrifuged and treated as per the aforementioned procedure. Enzymatic colorimetric assays were used to measure serum TG (Pureauto S TG-N, Sekisui Medical Co., Ltd., Tokyo, Japan), NEFA (NEFA-HR, Wako Pure Chemical Industries, Ltd., Osaka, Japan), TC (T-cho Determiner L TC II, Hitachi Chemical Diagnostics Systems Co., Ltd., Tokyo, Japan), LDL-C (Matabo Lead LDLC, Hitachi Chemical Diagnostics Systems Co., Ltd.), and HDL-C (Matabo Lead HDL-C, Hitachi Chemical Diagnostics Systems Co., Ltd.) and plasma glucose (GLU-HK(M), Shino-Test Corporation, Tokyo, Japan). Analyses for each participant were completed within the same run for each measure. Intra-assay coefficients of variation were $0.3 \%$ for 
TG, $0.8 \%$ for NEFA, $0.6 \%$ for TC, $0.8 \%$ for LDL-C, $1.2 \%$ for HDL-C and $0.4 \%$ for glucose.

\section{Sample size calculations and statistical analyses}

The sample size was set to 14 based on our pilot study and a previous study [6]. The validity of this sample size was examined by calculating the statistical power. The sample size was validated using L:M as the primary study outcome, using the same sample size calculation methods used in a previous crossover study [6]. Analyses were performed using G*Power 3.1.0 [22]. Based on a pilot study with ten participants, we calculated that an estimated total sample size of 14 was needed to provide $88 \%$ power to detect between-trial differences for two trials, with an alpha level set at 0.05 and a correlation of 0.5 . This sample size estimation was powered to detect an effect size of 0.91 (Cohen's $\mathrm{d}$ ), using a paired $\mathrm{t}$-test for comparison between trials. We also calculated the required sample size based on previous data [6]. The study reported within-subject effects (trial: glutamine vs. placebo; effect size, Cohen's $d=0.83$ for the plasma lactulose to rhamnose ratio) using glutamine versus the placebo response to a 60-min treadmill run [6]. The sample size was calculated to detect an effect size of 0.83 using a paired $t$-test for comparison between trials. For two trials with an alpha level set at 0.05 and a correlation of 0.5 , an estimated total sample size of 14 would provide $82 \%$ power to detect between-trial differences. Based on these calculations, we recruited 16 participants to allow for potential withdrawals. Data were analyzed using $R$ (version 3.4.3; R Foundation for Statistical Computing, Vienna, Austria), while linear mixed models were analyzed using the lme 4 and lmerTest libraries in R. The full analysis set was based on the intention-to-treat concept. A linear mixed model for repeated measures [trial (placebo or amino acids), time (treated as categorical with levels at 0,30,60, 120 and $240 \mathrm{~min}$ post-exercise], factors related to a crossover study design (i.e., term and sequence) as fixed factors, and participants as the random factor were used for each dependent variable. Statistical significance was accepted at a two-sided 5\% level. Results are reported as mean $\pm \mathrm{SD}$. However, in the linear mixed models for longitudinal data, the results for the trial are reported as coefficient \pm standard error $(\mathrm{SE})$, with the notation "coefficient $\pm \mathrm{SE}$ " to distinguish them from the mean $\pm \mathrm{SD}$.

\section{Results}

\section{Fasting biochemistry parameters}

Serum TG, NEFA, TC, LDL-C and HDL-C and plasma glucose concentrations measured on the morning of day 6 for each trial, are shown in Table 1. The fasting concentrations of all parameters did not differ between the trials.
Table 1 Fasting blood parameters measured on the morning of day 6 in the cystine and glutamine supplementation (Cys2/Gln) and placebo trials

\begin{tabular}{llll}
\hline & Cys2/Gln & Placebo & $P$ value \\
\hline TG $(\mathrm{mmol} / \mathrm{L})$ & $1.09 \pm 0.37$ & $1.08 \pm 0.35$ & 0.954 \\
NEFA $(\mathrm{mmol} / \mathrm{L})$ & $0.38 \pm 0.24$ & $0.45 \pm 0.33$ & 0.262 \\
TC $(\mathrm{mmol} / \mathrm{L})$ & $4.89 \pm 0.55$ & $4.82 \pm 0.60$ & 0.285 \\
LDL-C $(\mathrm{mmol} / \mathrm{L})$ & $2.83 \pm 0.45$ & $2.66 \pm 0.63$ & 0.098 \\
HDL-C $(\mathrm{mmol} / \mathrm{L})$ & $1.46 \pm 0.31$ & $1.53 \pm 0.24$ & 0.160 \\
Glucose $(\mathrm{mmol} / \mathrm{L})$ & $4.92 \pm 0.39$ & $4.77 \pm 0.40$ & 0.144 \\
\hline
\end{tabular}

Values are means \pm SD for $n=16$. Values were compared using paired $t$-tests

$T G$ triglycerides, NEFA non-esterified fatty acids, $T C$ total cholesterol, $L D L-C$ low-density lipoprotein cholesterol, $H D L-C$ high-density lipoprotein cholesterol. $P$ values were calculated by linear mixed effect models adjusted for crossover study design factors (i.e., term and sequence)

\section{Responses during running}

No significant differences were observed between the placebo, and cystine and glutamine supplementation trials regarding mean heart rate (placebo: $157 \pm 12$ beats/min; cystine and glutamine: $158 \pm 13$ beats/min, linear mixed model; $P=0.151$ ), relative exercise intensity (placebo: $78.3 \pm 5.8 \%$ of maximum oxygen uptake; cystine and glutamine: $78.9 \pm 5.7 \%$ of maximum oxygen uptake, linear mixed model; $P=0.168$ ), or peak rectal temperature (placebo $38.3 \pm 0.8{ }^{\circ} \mathrm{C}$; cystine and glutamine: $38.4 \pm 0.5^{\circ} \mathrm{C}$, linear mixed model; $P=0.686$ ).

\section{Plasma L:M}

The absolute plasma L:M ratio is shown in Fig. 2A. Plasma L:M was lower in the cystine and glutamine supplementation trial than in the placebo trial (linear mixed model, coefficient $\pm \mathrm{SE} ;-0.011 \pm 0.004, P=0.0090$ ). However, increased plasma L:M in the cystine and glutamine supplementation trial was observed at $120 \mathrm{~min}$ and $240 \mathrm{~min}$ after exercise. Given that this trend was also observed in the resting state (i.e., the baseline gastrointestinal permeability evaluation), an additional analysis was performed using the delta values (i.e., the change from the value in the resting state). In this additional analysis, plasma L:M was lower in the cystine and glutamine supplementation trials compared to that in the placebo trial (linear mixed model, coefficient \pm SE; $-0.011 \pm 0.004, P=0.0096)$ (Fig. $2 B$ ). 


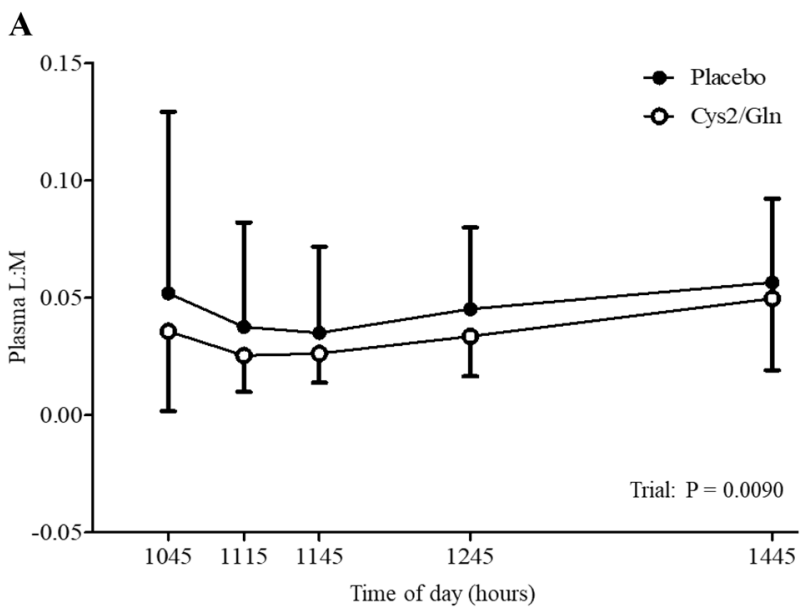

B

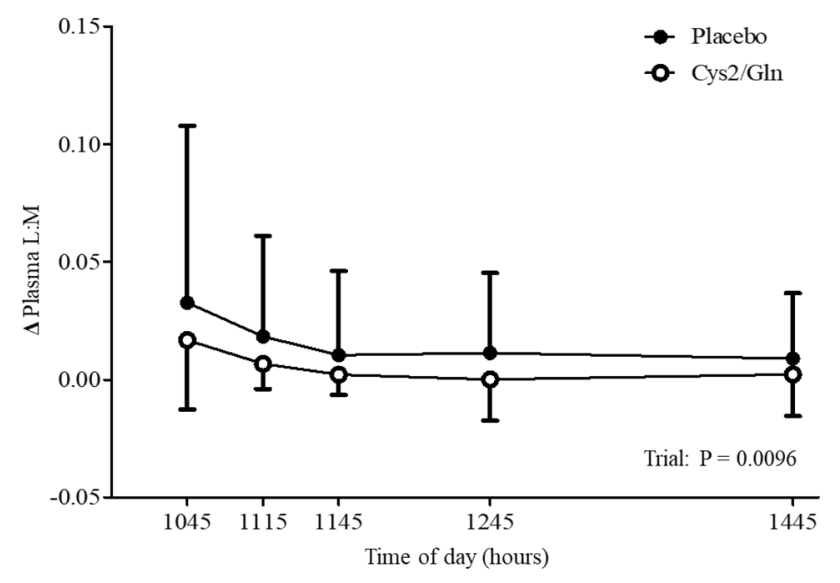

Fig. 2 Absolute (A) and delta (B) plasma lactulose to mannitol ratio (L:M) in the cystine and glutamine supplementation (Cys2/Gln) and placebo trials. Data are means \pm SD for $n=16$. Data were analyzed using a linear mixed model

\section{Plasma I-FABP}

Delta [by subtracting the value at each time point from the baseline value (i.e., 0900)] plasma I-FABP concentrations are shown in Fig. 3. Plasma I-FABP concentrations were lower in the cystine and glutamine supplementation trial than in the placebo trial (linear mixed model, coefficient $\pm \mathrm{SE}[\mathrm{pg} / \mathrm{mL}] ;-195.3 \pm 65.7, P=0.0035)$.

\section{Discussion}

The present study demonstrated that compared with ingestion of maltodextrin, oral cystine and glutamine supplementation attenuated the exercise-induced increase in plasma L:M and I-FABP concentrations in young active men. A small amount of cystine $(0.69 \mathrm{~g} /$ day from days $1-5$, and $0.23 \mathrm{~g} /$ day on day 6$)$ and glutamine (3.00 g/day from days

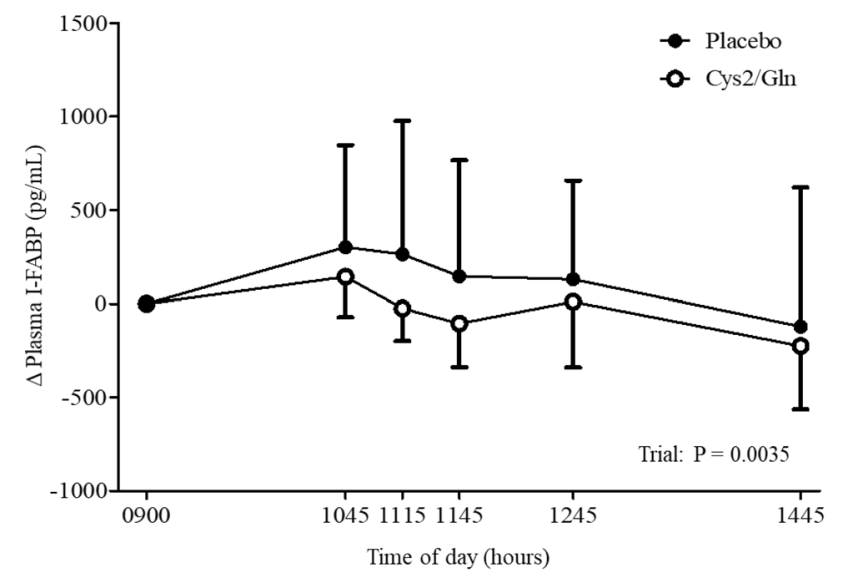

Fig. 3 Delta plasma intestinal fatty acid-binding protein (I-FABP) in the cystine and glutamine supplementation (Cys2/Gln) and placebo trials. Data are means \pm SD for $n=16$. Data were analyzed using a linear mixed model

$1-5$, and $1.00 \mathrm{~g} /$ day on day 6) were used in the present study, compared with the amounts of amino acids used in previous studies [i.e., ranging from $0.25 \mathrm{~g} / \mathrm{kg}$ fat-free mass (FFM) to $0.9 \mathrm{~g} / \mathrm{kg} \mathrm{FFM}$ (or /day)] [6, 7, 9, 23]. Our findings may, therefore, underscore the practical importance of nutritional strategies to reduce gastrointestinal problems in real-life settings where athletes, endurance athletes in particular, often experience such complications, both during and after prolonged strenuous exercise [24].

Previous studies investigated the potential role of nutritional supplement interventions for the prevention or attenuation of exercise-induced gastrointestinal permeability markers in response to exercise under conditions of exercise-heat stress or field competitive events in humans-these were conducted with various supplements, including antioxidants, amino acids (i.e., L-arginine and glutamine), bovine colostrum, curcumin, and probiotics (for a review of these, see Reference [5]). Three of these studies reported reductions in the markers of gastrointestinal permeability [6, 9, 25]. Of these three studies, two studies used glutamine and were characterized by a similar duration (i.e., for 7 days or immediately pre-exercise) and/or amount of supplements $(0.9 \mathrm{~g} /$ $\mathrm{kg} \mathrm{FFM/day} \mathrm{or} 0.25,0.5$ and $0.9 \mathrm{~g} / \mathrm{kg}$ FFM) used in the present study $[6,9]$. Furthermore, regarding the dosage of glutamine and the magnitude of response in gastrointestinal permeability markers after exercise, one study has reported that $0.9 \mathrm{~g} / \mathrm{kg}$ FFM/day of glutamine administered for 7 days attenuated the increases in gastrointestinal permeability by approximately $55 \%$ compared to placebo [6]. Another study, in which oral glutamine was consumed $2 \mathrm{~h}$ before running, has also reported reduced gastrointestinal permeability by approximately $25 \%$ with $0.25 \mathrm{~g} / \mathrm{kg}$ FFM of glutamine and by approximately $40 \%$ with $0.9 \mathrm{~g} / \mathrm{kg}$ FFM of glutamine at $60 \mathrm{~min}$ post-exercise compared to placebo [9]. In the present 
study, increased gastrointestinal permeability (i.e., a mean of $4 \mathrm{~h}$ after exercise) was found to be attenuated by approximately $66 \%$ with oral cystine and glutamine supplementation compared to the placebo trial. Also, it is worth noting that these two studies $[6,9]$ conducted exercises at a relatively higher temperature $\left(30^{\circ} \mathrm{C}\right)$ than the present study $\left(25^{\circ} \mathrm{C}\right)$. Nonetheless, given that there is a positive relationship between increased core body temperature (induced by intense and prolonged exercise in a hyperthermic environment) and increased gastrointestinal permeability [1, 3], the present study also observed an increase in core body temperature to $38{ }^{\circ} \mathrm{C}$ (i.e., on average in both trials). This effectively attenuated the increases in gastrointestinal permeability and damage by oral cystine and glutamine supplementation. Indeed, our findings are consistent with those of previous studies [21,26-28] where the environmental conditions and/or exercise volumes were similar to those of the present study although various supplements (i.e., bovine colostrum, zinc and probiotics) were used in these previous studies. However, inconsistent results have also been reported regarding its effectiveness in lowering gastrointestinal permeability and intestinal cellular damage [29-31]. The duration of exercise, dosage of the saccharide used in a permeability test, nutritional status before the experiment (i.e., a fasted or fed state), timing of a saccharide drink ingestion (i.e., before or after exercise) and environmental conditions are possible reasons for the inconsistent findings among studies as these are confounding factors affecting the gastrointestinal permeability and damage [3]. Additional research with a standardized protocol is required to elucidate the impact of supplementation on gastrointestinal permeability and damage in response to exercise in various environments.

The mechanisms underlying the exercise-induced increase in gastrointestinal permeability and intestinal cellular damage are unclear, but are likely mediated by an increase in core temperature during or shortly after strenuous exercise, and/or a reduced total splanchnic perfusion, causing gastrointestinal ischemia [3, 4]. Glutamine intake in response to running (i.e., $60 \mathrm{~min}$ at $65-70 \%$ of maximal oxygen uptake) has been shown to stimulate heat shock protein 70 and nuclear factor of kappa light polypeptide gene enhancer in B-cells inhibitor alpha in human peripheral blood mononuclear cells, which leads to inactivation of the nuclear translocation of nuclear factor- $\mathrm{\kappa B}$ proinflammatory pathway [6]. A recent in vitro study also suggested that cystine reduces barrier dysfunction caused by oxidative stress and improves intestinal barrier function [13]. It is not possible to determine which of these mechanisms operated in the present study, but mixed supplementation of cystine and glutamine may have contributed to facilitating these mechanisms and attenuating exercise-induced increases in plasma L:M and I-FABP concentrations. Despite the effectiveness of supplementation for the prevention of increased gastrointestinal permeability and damage in response to exercise, though not all [23], glutamine supplementation did not appear to influence subjective symptoms of gastrointestinal discomfort in response to heat [9]. Therefore, further studies are needed to determine the effect of supplementation, if any, on the subjective symptoms of gastrointestinal discomfort in a real-world setting (i.e., repeated exercise training).

The strength of the present study includes the protective effect of intestinal permeability and damage were observed at relatively low mixed amino acid doses. Indeed, most previous studies using glutamine as a nutritional intervention have employed relatively large doses of glutamine (range from $0.25 \mathrm{~g} / \mathrm{kg}$ FFM to $0.9 \mathrm{~g} / \mathrm{kg}$ FFM/day) [5]. Thus, our supplementation dose is more accessible to consume on a daily basis. There are limitations to the present study that should be addressed. Our findings were not able to provide potential mechanisms for the attenuation of the exerciseinduced increase in plasma L:M and I-FABP concentrations since the present study was not focused on or designed with a mechanistic intent. In addition, the present study was designed to explore the effect of prior amino acid supplementation on markers of gastrointestinal permeability and damage in response to an acute bout of prolonged strenuous exercise in active men. Thus, the effectiveness of post-exercise supplementation on exercise-induced gastrointestinal syndrome in trained athletes is not known. Future research is required for a longer observational period than the protocol used in the present study to observe the favourable effects, if any, on post-exercise nutritional strategies in the presence of increased gastrointestinal permeability and damage or gastrointestinal syndrome in this population [3].

\section{Conclusion}

In conclusion, oral cystine and glutamine supplementation attenuated L:M and plasma I-FABP concentrations in response to $1 \mathrm{~h}$ of strenuous running in young active men, suggesting a transient reduction in gastrointestinal permeability and damage via amino acid supplementation. These findings require further investigation over a longer period with a more practical setting in athletes who experience gastrointestinal problems through habitual exercise training.

Acknowledgements All authors would like to thank the researchers of Ajinomoto Co., Inc., Takuya Toyoda, Ikuko Sasahara, Michihiro Takada and Wataru Sato who contributed to the development of the study.

Author contributions $\mathrm{YT}$ and $\mathrm{AH}$ performed the data interpretation and wrote the manuscript. $\mathrm{MH}$ and $\mathrm{YH}$ supervised the data collection and assisted with the analytical measurements. MO and TY designed the study. ST performed the statistical analysis. KS advised the data 
interpretation to YT and revised the manuscript. MM conceived the study and took the lead in writing the manuscript. All authors approved the final version of the manuscript.

Funding This study was funded by Ajinomoto Co., Inc., Japan.

Data availability Some or all data sets generated during and/or analyzed during the present study are not publicly available but are available from the corresponding author on reasonable request.

\section{Declarations}

Conflict of interest MM has received research funding from Ajinomoto Co., Inc. MO, ST and TY are employees of Ajinomoto Co., Inc. For the remaining authors, none were declared conflicts of interest. All authors declare that the findings of the present study are presented clearly, honestly and without fabrication, falsification, or inappropriate data manipulation.

Ethical standards All procedures performed in the study involving human participants were in accordance with the ethical standards of the institutional and national research committee, and with the 1964 Helsinki declaration and its later amendments.

Ethics approval This study was conducted under approval from the Human Research Ethical Advisory Committee of Waseda University (Approval number: 2018-270),

Consent to participate Informed consent was obtained from all participants included in this study.

Consent for publication Not applicable.

Open Access This article is licensed under a Creative Commons Attribution 4.0 International License, which permits use, sharing, adaptation, distribution and reproduction in any medium or format, as long as you give appropriate credit to the original author(s) and the source, provide a link to the Creative Commons licence, and indicate if changes were made. The images or other third party material in this article are included in the article's Creative Commons licence, unless indicated otherwise in a credit line to the material. If material is not included in the article's Creative Commons licence and your intended use is not permitted by statutory regulation or exceeds the permitted use, you will need to obtain permission directly from the copyright holder. To view a copy of this licence, visit http://creativecommons.org/licenses/by/4.0/.

\section{References}

1. Pires W, Veneroso CE, Wanner SP, Pacheco DAS, Vaz GC, Amorim FT, Tonoli C, Soares DD, Coimbra CC (2017) Association between exercise-induced hyperthermia and intestinal permeability: a systematic review. Sports Med 47(7):1389-1403. https:// doi.org/10.1007/s40279-016-0654-2

2. Lim CL, Suzuki K (2017) Systemic inflammation mediates the effects of endotoxemia in the mechanisms of heat stroke. Biol Med 9(1):8-11

3. Chantler S, Griffiths A, Matu J, Davison G, Jones B, Deighton K (2021) The effects of exercise on indirect markers of gut damage and permeability: a systematic review and meta-analysis. Sports Med 51(1):113-124. https://doi.org/10.1007/s40279-020-01348-y
4. Costa RJS, Snipe RMJ, Kitic CM, Gibson PR (2017) Systematic review: exercise-induced gastrointestinal syndrome-implications for health and intestinal disease. Aliment Pharmacol Ther 46(3):246-265. https://doi.org/10.1111/apt.14157

5. Costa RJS, Gaskell SK, McCubbin AJ, Snipe RMJ (2020) Exertional-heat stress-associated gastrointestinal perturbations during olympic sports: management strategies for athletes preparing and competing in the 2020 tokyo olympic games. Temperature 7(1):58-88. https://doi.org/10.1080/23328940.2019.1597676

6. Zuhl MN, Lanphere KR, Kravitz L, Mermier CM, Schneider S, Dokladny K (1985) Moseley PL (2014) Effects of oral glutamine supplementation on exercise-induced gastrointestinal permeability and tight junction protein expression. J Appl Physiol 116(2):183191. https://doi.org/10.1152/japplphysiol.00646.2013

7. Zuhl M, Dokladny K, Mermier C, Schneider S, Salgado R, Moseley P (2015) The effects of acute oral glutamine supplementation on exercise-induced gastrointestinal permeability and heat shock protein expression in peripheral blood mononuclear cells. Cell Stress Chaperones 20(1):85-93. https://doi.org/10. 1007/s12192-014-0528-1

8. Zuhl M, Schneider S, Lanphere K, Conn C, Dokladny K, Moseley $\mathrm{P}$ (2014) Exercise regulation of intestinal tight junction proteins. Br J Sports Med 48(12):980-986. https://doi.org/10.1136/ bjsports-2012-091585

9. Pugh JN, Sage S, Hutson M, Doran DA, Fleming SC, Highton J, Morton JP, Close GL (2017) Glutamine supplementation reduces markers of intestinal permeability during running in the heat in a dose-dependent manner. Eur $\mathbf{J}$ Appl Physiol 117(12):2569-2577. https://doi.org/10.1007/ s00421-017-3744-4

10. Rimaniol AC, Mialocq P, Clayette P, Dormont D, Gras G (2001) Role of glutamate transporters in the regulation of glutathione levels in human macrophages. Am J Physiol Cell Physiol 281(6):C1964-C1970. https://doi.org/10.1152/ajpcell.2001.281.6. C1964

11. Circu ML, Aw TY (2011) Redox biology of the intestine. Free Radic Res 45(11-12):1245-1266. https://doi.org/10.3109/10715 762.2011.611509

12. Tanaka KA, Kurihara S, Shibakusa T, Chiba Y, Mikami T (2015) Cystine improves survival rates in a LPS-induced sepsis mouse model. Clin Nutr 34(6):1159-1165. https://doi.org/10.1016/j.clnu. 2014.11.014

13. Hasegawa T, Mizugaki A, Inoue Y, Kato H, Murakami H (2021) Cystine reduces tight junction permeability and intestinal inflammation induced by oxidative stress in Caco- 2 cells. Amino Acids 53(7):1021-1032. https://doi.org/10.1007/s00726-021-03001-y

14. World Health Organization. Global Recommendations on physical activity for health. Geneva (Switzerland): World Health Organization; 2010. (cited 4 July 2021). Available from: https://apps.who. int/iris/handle/10665/44399

15. Taylor HL, Buskirk E, Henschel A (1955) Maximal oxygen intake as an objective measure of cardio-respiratory performance. J Appl Physiol 8(1):73-80. https://doi.org/10.1152/jappl.1955.8.1.73

16. Borg GA (1973) Perceived exertion: a note on "history" and methods. Med Sci Sports 5(2):90-93. https://doi.org/10.1249/00005 768-197300520-00017

17. Sasaki E, Umeda T, Takahashi I, Arata K, Yamamoto Y, Tanabe M, Oyamada K, Hashizume E, Nakaji S (2013) Effect of glutamine supplementation on neutrophil function in male judoists. Luminescence 28(4):442-449. https://doi.org/10.1002/bio.2474

18. Kurihara S, Yoshida S, Sukegawa E, Yoshimura S, Uchida H, Maeda K, Yamamoto T (2008) Evaluation of safety of long-term and excessive intake of 1-cystine and 1-theanine in healthy adult subjects. Seikatsu Eisei 52(4):229-236 (in Japanese). https://doi. org/10.11468/seikatsueisei.52.229 
19. Gervasoni J, Primiano A, Graziani C, Scaldaferri F, Gasbarrini A, Urbani A, Persichilli S (2018) Validation of UPLC-MS/MS Method for determination of urinary lactulose/mannitol. Molecules 23(10):2705. https://doi.org/10.3390/molecules23102705

20. Bioanalytical Method Validation in Japan. Guideline on bioanalytical method validation in pharmaceutical development. Kawasaki (Japan): National Institute of Health Sciences; 2013. (cited 4 July 2021)

21. March DS, Marchbank T, Playford RJ, Jones AW, Thatcher R, Davison G (2017) Intestinal fatty acid-binding protein and gut permeability responses to exercise. Eur J Appl Physiol 117(5):931941. https://doi.org/10.1007/s00421-017-3582-4

22. Faul F, Erdfelder E, Lang AG, Buchner A (2007) G*Power 3: A flexible statistical power analysis program for the social, behavioral, and biomedical sciences. Behav Res Methods 39(2):175-191. https://doi.org/10.3758/bf03193146

23. Osborne JO, Stewart IB, Beagley KW, Borg DN, Minett GM (2019) Acute glutamine supplementation does not improve 20-km self-paced cycling performance in the heat. Eur J Appl Physiol 119(11-12):2567-2578. https://doi.org/10.1007/ s00421-019-04234-2

24. De Oliveira EP, Burini RC, Jeukendrup A (2014) Gastrointestinal complaints during exercise: Prevalence, etiology, and nutritional recommendations. Sports Med 44(Suppl 1):S79-85. https://doi. org/10.1007/s40279-014-0153-2

25. Szymanski MC, Gillum TL, Gould LM, Morin DS (1985) Kuennen MR (2018) Short-term dietary curcumin supplementation reduces gastrointestinal barrier damage and physiological strain responses during exertional heat stress. J Appl Physiol 124(2):330-340. https://doi.org/10.1152/japplphysiol.00515.2017
26. March DS, Jones AW, Thatcher R, Davison G (2019) The effect of bovine colostrum supplementation on intestinal injury and circulating intestinal bacterial DNA following exercise in the heat. Eur J Nutr 58(4):1441-2145. https://doi.org/10.1007/ s00394-018-1670-9

27. Davison G, Marchbank T, March DS, Thatcher R, Playford RJ (2016) Zinc carnosine works with bovine colostrum in truncating heavy exercise-induced increase in gut permeability in healthy volunteers. Am J Clin Nutr 104(2):526-536. https://doi.org/10. 3945/ajen.116.134403

28. Mooren FC, Maleki BH, Pilat C, Ringseis R, Eder K, Teschler M, Krüger K (2020) Effects of Escherichia coli strain Nissle 1917 on exercise-induced disruption of gastrointestinal integrity. Eur J Appl Physiol 120(7):1591-1599. https://doi.org/10.1007/ s00421-020-04382-w

29. Shing CM, Peake JM, Lim CL, Briskey D, Walsh NP, Fortes MB, Ahuja KD, Vitetta L (2014) Effects of probiotics supplementation on gastrointestinal permeability, inflammation and exercise performance in the heat. Eur J Appl Physiol 114(1):93-103. https:// doi.org/10.1007/s00421-013-2748-y

30. Van Wijck K, Wijnands KAP, Meesters DM, Boonen B, van Loon LJ, Buurman WA, Dejong CH, Lenaerts K, Poeze M (2014) L-citrulline improves splanchnic perfusion and reduces gut injury during exercise. Med Sci Sports Exerc 46(11):2039-2046. https:// doi.org/10.1249/MSS.0000000000000332

31. West NP, Pyne DB, Cripps AW, Christophersen CT, Conlon MA, Fricker PA (2012) Gut balance, a synbiotic supplement, increases fecal Lactobacillus paracasei but has little effect on immunity in healthy physically active individuals. Gut Microbes 3(3):221-227. https://doi.org/10.4161/gmic.19579 